\title{
A Case of Concomitant Obstructive Sleep Apnea and Non- Alcoholic Steatohepatitis Treated With CPAP Therapy
}

\author{
Bharat Bajantria, c, Dmitry Lvovskya, b
}

\begin{abstract}
Obstructive sleep apnea syndrome is a disorder of sleep breathing that is a result of recurrent and intermittent hypoxia during sleep induced by the repeated partial or complete collapse of the upper airway, eventually causing chronic intermittent hypoxia. Non-alcoholic fatty liver disease is divided into non-alcoholic fatty liver and non-alcoholic steatohepatitis. Animal and human studies showed that obesity is associated with chronic liver hypoxia, even in the presence of systemic normoxia causing inflammation and release of cytokines. A "two-hit" model has been proposed. The first hit is characterized by insulin resistance and excess hepatic lipid accumulation secondary to abnormal fatty acid metabolism. Oxidative stress and inflammation are thought to comprise the second hit. Gold standard for the diagnosis of non-alcoholic steatohepatitis is a liver biopsy. Many clinical scores and noninvasive tools are used for the diagnosis of non-alcoholic steatohepatitis. Conservative management with lifestyle modifications including diet, exercise and weight loss remains the therapy of choice today. We present a case report of a 39-year-old man who was diagnosed with concomitant non-alcoholic steatohepatitis and severe obstructive sleep apnea. He was started treatment with continuous positive airway pressure and demonstrated excellent adherence to therapy for 6 years, with concomitant obstructive sleep apnea and non-alcoholic steatohepatitis which reversed with prolonged optimal continuous positive airway pressure therapy. Physical examination remained unremarkable except for morbid obesity. His abdominal girth, as well as body mass index, remained unchanged. After 6 years of optimal continuous positive airway pressure therapy, liver enzymes and relevant lipid panel normalized, suggesting reversal of non-alcoholic steatohepatitis.
\end{abstract}

Keywords: Obstructive sleep apnea; Fatty liver; Non-alcoholic liver disease; Non-alcoholic steatohepatitis; Continuous positive airway pressure; Metabolic syndrome

Manuscript submitted May 1, 2018, accepted May 9, 2018

aDivision of Pulmonary Critical Care, Department of Medicine, Bronx Care Health System, Bronx, NY 10457, USA

bDivision of Pulmonary and Critical Care Medicine, Bronx Lebanon Hospital Center, Bronx, NY 10457, USA

${ }^{\mathrm{c} C}$ Corresponding Author: Bharat Bajantri, Division of Pulmonary Critical Care, Department of Medicine, Bronx Care Health System, Bronx, NY 10457, USA. Email: bharatbajantri@gmail.com

doi: https://doi.org/10.14740/gr1033w

\section{Introduction}

Obstructive sleep apnea (OSA) syndrome is a disorder of sleep breathing that is a result of recurrent and intermittent hypoxia during sleep induced by the repeated partial or complete collapse of the upper airway, eventually causing chronic intermittent hypoxia (CIH) [1]. The adult OSA task force of the American Academy of Sleep Medicine defines clinical OSA characterized by the occurrence of daytime sleepiness, loud snoring, witnessed breathing interruptions or awakenings due to gasping or choking in the presence of at least five obstructive respiratory events - apneas, hypopneas per hour of sleep index (AHI). The presence of AHI of 15 or greater in the absence of sleep-related symptoms is also sufficient for the diagnosis of OSA due to the greater association of this severity of obstruction with important consequences such as increased cardiovascular disease risk [2].

Arbitrary criteria to define OSA syndrome have led to a wide range of incidence of OSA that has been reported from $4 \%$ to more than $60 \%$ among the adult population [3]. Prevalence of certain risk factors like obesity (body mass index (BMI) $>35 \mathrm{~kg} / \mathrm{m}^{2}$ ), congestive heart failure, atrial fibrillation, treatment-refractory hypertension, type 2 diabetes, nocturnal dysrhythmias, stroke, pulmonary hypertension, high-risk driving populations and preoperative bariatric patients increase the incidence much more than the general population [2].

Non-alcoholic fatty liver disease (NAFLD) is divided into non-alcoholic fatty liver (NAFL) and non-alcoholic steatohepatitis (NASH). They are differentiated from each other by the histological presence of cellular injury and fibrosis in NASH and its absence in NAFLD. Macrovesicular steatosis with lobular and periportal inflammation may be seen in both [4].

Evidence suggests a strong association of metabolic risk factors like obesity, type 2 diabetes mellitus, elevated triglyceride levels, cardiovascular disease and NAFLD with OSA $[1,5-10]$. Prevalence of NAFLD is around $30 \%$ in the general population, but it doubles among patients with diabetes or obesity [11]. Studies show that OSA syndrome can cause intrinsic liver injury and lead to NAFLD $[12,13]$. This can happen independent of obesity. Although there are conflicting results on the effect of continuous positive airway pressure (CPAP) on liver enzymes (used as surrogates for the intrinsic function of the liver in NAFLD), the majority of the studies were confounded, non-randomized and small [14-17]. This case presents NAFLD associated with OSA syndrome, which reversed 
Table 1. Patient's Compliance Data for the Last 1 Year

\begin{tabular}{ll}
\hline Days used in the $\mathbf{3 6 5}$ days & $\mathbf{3 6 5}$ days \\
\hline PAP device & Automatic positive airway pressure \\
PAP pressures & $10-18 \mathrm{~cm}$ \\
Average number of hours used per day & $7 \mathrm{~h} 32 \mathrm{~min}$ \\
Average leak & $3.7 \mathrm{~L} / \mathrm{M}$ \\
Residual AHI & 0.7 \\
\hline
\end{tabular}

with optimal CPAP therapy over a prolonged duration of time.

\section{Case Report}

The patient was a 39-year-old Hispanic man with medical co-morbidities of morbid obesity, diabetes mellitus, hypertension, hypercholesterolemia and liver dysfunction. He was initially seen in our sleep clinic for symptoms of loud snoring, witnessed apnea, daytime fatigue and hypersomnolence. His Epworth sleepiness scale was 15 . He underwent in-lab polysomnography (PSG) that revealed severe OSA with AHI of 50 and the lowest oxygen saturation detected during the study was $63 \%$. During the same PSG, he was successfully titrated to CPAP of $16 \mathrm{~cm} \mathrm{H}_{2} \mathrm{O}$. Subsequently, his adherence to CPAP therapy was assessed at regular intervals demonstrating excellent adherence. An example of his compliance data for the last year is shown in Table 1.

His family history was unremarkable for any liver or sleep disorders. He denied toxic habits including consumption of tobacco, alcohol or any other recreational drugs. He did not receive any blood transfusions. The abdominal girth, as well as $\mathrm{BMI}$, remained unchanged in the range of $39.7-40.6 \mathrm{~kg} / \mathrm{m}^{2}$ for more than 10 years. He felt that his overall health and exercise tolerance have improved after starting CPAP therapy.

Physical examination remained unremarkable except for morbid obesity with BMI $40 \mathrm{~kg} / \mathrm{m}^{2}$. Initial laboratory tests performed between September 2011 and June 2012 are illustrated in Table 2.

Table 2. Laboratory Values

\begin{tabular}{llll}
\hline Hematology & Value & Biochemistry & Value \\
\hline White cell count $(\mathrm{k} / \mu \mathrm{L})$ & 9.2 & Creatinine $(\mathrm{mg} / \mathrm{dL})$ & 0.8 \\
Neutrophils $(\%)$ & 58.7 & Blood urea nitrogen $(\mathrm{mg} / \mathrm{dL})$ & 11 \\
Lymphocyte $(\%)$ & 32 & Serology & Negative \\
Red blood cell $(\mathrm{mil} / \mu \mathrm{L})$ & 5.51 & ANA & Negative \\
Hemoglobin $(\mathrm{g} / \mathrm{dL})$ & 17.2 & AMA & Negative \\
Hematocrit $(\%)$ & 51.6 & Hepatitis B surface antigen & Negative \\
Platelets $(\mathrm{k} / \mu \mathrm{L})$ & 205 & Hepatitis B core antibody & Negative \\
Activated partial thromboplastin time $(\mathrm{s})$ & 38.7 & Hepatitis C virus antibody & Thyroid function \\
Prothrombin time $(\mathrm{s})$ & 10.9 & TSH $(\mathrm{mIU} / \mathrm{L})$ & 1.02 \\
INR & 1.0 & FT3 (ng/dL) & 166 \\
Ferritin $(\mathrm{ng} / \mathrm{dL})$ & 587 & FT4 $(\mathrm{ng} / \mathrm{dL})$ & 1.25 \\
\hline
\end{tabular}

Figure 1 shows the trends of liver enzymes and lipid panel.

Ultrasonography of the liver showed deep-echo attenuation with hepatomegaly, highly suggestive of fatty infiltration of the liver. Computed tomography (CT) of the abdomen concurred with these findings. Following this, he underwent CT-guided liver biopsy that demonstrated moderate macrovesicular steatosis with inflammation, hepatocyte ballooning degeneration and portal fibrosis. On the basis of this, he was diagnosed with NASH stage 2.

After approximately 1 year of CPAP therapy, liver enzyme levels had decreased. Finally, at 6 years liver enzymes and relevant lipid panel normalized as graphically represented in Figure 1. Serum gamma-glutamyl transferase levels decreased from $68 \mathrm{IU} / \mathrm{L}$ to normal levels over 6-year period. Other pertinent labs including that of HbA1c and HDL also improved and normalized. His lifestyle remained unchanged since he was diagnosed with OSA. There were no additional medications used for the treatment of NASH in this patient. Our case stands out for its prolonged CPAP therapy follow-up with the evidence of optimal compliance. As such, it can potentially reverse NASH, which is contradictory to what was found in few available randomized control trials (RCTs), which were characterized by small size, poor design and short duration of therapy [14-18].

\section{Discussion}

The liver receives its blood supply from the highly oxygenated 


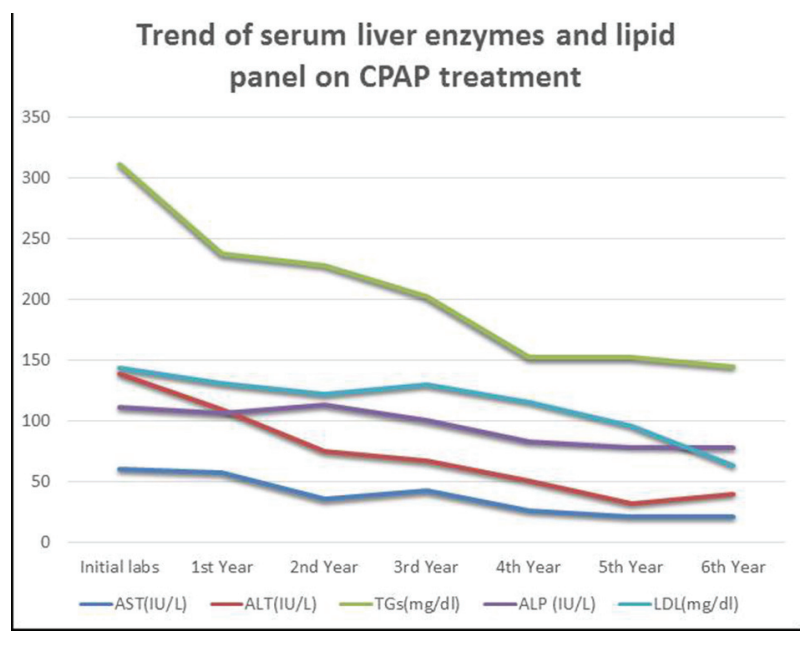

Figure 1. Trends of liver enzymes and lipid panel. AST, aspartate transaminase; ALT, alanine aminotransferase; ALP, alkaline phosphatase; TGs, triglycerides; LDL, low-density lipoprotein.

hepatic artery and a poorly oxygenated portal vein, thus causing an oxygen gradient in the hepatic lobule. The partial pressure of oxygen in the periportal blood is $60-65 \mathrm{~mm} \mathrm{Hg}$ and that in the peri-central venular blood is $30-35 \mathrm{~mm} \mathrm{Hg}$. This makes liver sensitive to hypoxia and different parts of the hepatic lobule respond differently to hypoxia. Periportal hepatocytes play a predominant role in oxidative energy metabolism, glucose production, and urea and bile biosynthesis. Peri-central venular hepatocytes take up roles in glucose uptake and storage as glycogen, glutamine formation and xenobiotic metabolism $[19,20]$. Animal studies have demonstrated that hypoxia alone aggravates and accelerates the progression of NASH by up-regulating the expression of lipogenic genes, by down-regulating genes involved in lipid metabolism and by decreasing insulin sensitivity [21]. Experiments on mice revealed liver to be most sensitive for acute intermittent hypoxia causing reciprocal oxygen oscillations when compared to other tissues. Acute intermittent hypoxia increases the oxidative stress and adipose inflammation irrespective of body fat content. Animal and human studies showed that obesity is associated with chronic liver hypoxia, even in the presence of systemic normoxia causing inflammation and release of cytokines [22]. On this basis, it is speculated that OSA syndrome might synergize with obesity to accelerate the process of hepatic dysfunction and death, aggravating liver and metabolic disease. Nocturnal hypoxia has been linked to decreasing adiponectin level that is implicated in cardiovascular diseases and OSA syndrome $[23,24]$. Hypoxia-induced release of cytokines and reactive oxygen species increases the release of nuclear factor kappa $\mathrm{B}(\mathrm{NF}-\mathrm{kB})$, which is implicated in OSA syndrome. $\mathrm{CIH}$ has been associated with endoplasmic reticulum stress that leads to accumulation of unfolded proteins at the molecular level [25].

In recent years, many observational human studies evaluating the relationship of OSA syndrome and NAFLD have been published. Heterogeneity in terms of definitions of NAFLD, as well as cutoff points for acute intermittent hypoxia to characterize OSA syndrome among these observational studies, limits its clinical applicability. Obesity and lack of liver biopsies among all subjects were the biggest confounding factors among most of these studies preventing us from drawing independent association of OSA syndrome and NAFLD $[12,26-32]$. Tatsumi et al evaluated 83 non-obese patients and concluded that oxygen desaturation during sleep is a risk factor for progressive liver disease from the accumulation of fat. They, however, employed serum type III procollagen as a marker of liver fibrosis. Another study used liver ultrasound for evaluation of NAFLD among 106 patients with severe OSA syndrome and found NAFLD in two-thirds of patients, and among them, $70 \%$ had severe OSA syndrome [27]. Polotsky et al further showed that nocturnal oxygen desaturation contributed to insulin resistance and to different histological features with varying degrees of liver injury, including hepatocyte ballooning, inflammation and fibrosis, but failed to show hepatic steatosis per se. On multivariate analysis, AHI, oxygen desaturation index (ODI), lowest desaturation values and percentage of sleep duration with $\mathrm{SpO}_{2}<90 \%$ independently predicted NAFLD after adjustment for BMI, weight and insulin resistance. Furthermore, the best predictor of the severity of NAFLD was the duration of hypoxia during sleep [32]. Another observational study by Aron-Wisnewsky et al found that when ODI was used to stratify severity of OSA syndrome among 101 morbidly obese patients, the prevalence of NAFLD was $77 \%$. Among patient with NAFLD, close to $90 \%$ had evidence of fibrosis on histology. In this study, ODI was strongly associated with fibrosis and NAFLD activity score (NAS) [13, 33].

Pathogenesis of NAFLD is poorly understood. A "two-hit" model has been proposed. The first hit is characterized by insulin resistance and excess hepatic lipid accumulation secondary to abnormal fatty acid metabolism. Oxidative stress and inflammation are thought to comprise the second hit [1]. RCTs and meta-analysis confirm that CPAP treatment for more than 3 months has shown to improve insulin resistance $[14,18,34$, 35]. Decrease in adiponectin level and increase in leptin level are associated with OSA syndrome. Visceral fat even in nonobese patients with OSA syndrome is associated with insulin resistance. There is some evidence to suggest that their levels are somewhat corrected with prolonged CPAP therapy [36-40]. $\mathrm{CIH}$, which is the distinctive feature of OSA syndrome, is proposed to stimulate peripheral lipolysis and inhibit lipoprotein clearance [1]. Lin et al reported a meta-regression analysis with almost 2,000 patients and showed that CPAP therapy improved cholesterol and triglyceride levels [41]. Another meta-analysis with only RCTs also showed similar results [42].

$\mathrm{CIH}$ triggers oxidative stress that leads to increased release of radical oxygen species like superoxide. There are other proinflammatory markers like C-reactive protein, interleukin-8 and tumor necrosis factor alpha. CPAP therapy has shown to decrease these levels [43-46]. It has been suggested that, because all these studies did not exclusively include NAFLD patients, CPAP therapy is potentially a preventive measure for liver disease among patients with normal liver function at baseline [1].

NAFLD is characterized by a spectrum of histological findings from simple steatosis to steatosis with necroinflammation (also called NASH). NAFLD can be diagnosed by imaging studies such as ultrasound, CT or magnetic resonance 
imaging [47]. Prevalence of NASH ranges from 5\% to $33 \%$ in the general population to $90 \%$ among the morbidly obese $[48,49]$. Historically, the gold standard for the diagnosis of NASH is a liver biopsy. Many clinical scores like NAS and SAF (steatosis, activity and fibrosis) are used for the diagnosis of NAFLD $[50,51]$. Non-invasive tools like fatty liver index, serum algorithm and elastography for diagnosis of NASH have been recently validated [51-56]. The clinical significance of NASH is that it is associated with varying degrees of fibrosis, extremes of which can progress to cirrhosis and hepatocellular carcinoma $[57,58]$. In our patient, NASH was diagnosed by liver biopsy.

OSA syndrome must be suspected in patients with excessive daytime sleepiness, fatigue, non-refreshing sleep, nocturia, morning headache, irritability and memory loss [59-61]. Only about $50 \%$ of patient with OSA syndrome are obese. Up to $25 \%$ of patients with sleep-disordered breathing present neither objective or subjective symptoms of OSA syndrome $[62,63]$. Similarly, patients with NAFLD are largely asymptomatic with the common presentation of incidental abnormal liver function tests. They are, however, considered to be part of the metabolic syndrome with obesity and insulin resistance. They are also associated with diabetes mellitus and hypertension [64].

Clinical features like neck circumference, Mallampati score, use of questionnaires like Epworth sleepiness scale, Berlin questionnaire, STOPBANG (snoring, tiredness, observed apnea, hypertension, BMI, age, neck circumference and gender), etc., have shown $80-90 \%$ sensitivity but only $35 \%$ specificity. The gold standard for diagnosis of OSA syndrome is inlab PSG; however, in the recent past, more evidence suggests that less expensive and less elaborate home sleep testing may be just as good in certain situations [65-67]. CPAP is the first choice of treatment across all grades of severity of OSA syndrome and is recommended to be offered to all patients [68].

There are no FDA approved drugs for NASH. Conservative management with lifestyle modifications including diet, exercise and weight loss remains the therapy of choice today. Many therapeutic trials have shown little success. Some of the drugs that have been tested include liver-directed therapy (vitamin E, pentoxyphylline, pioglitazone, etc.) for NAFLD [69-71]. Hence, in this current scenario, we are desperate to explore and find more definitive treatment. Treatment of OSA syndrome with CPAP has demonstrated a decrease in most of the metabolic risk factors [72-75]. CPAP treatment directed for OSA syndrome is thought to negate some of the downfalls of NAFLD. RCTs with sham controls to evaluate the effect of CPAP on liver function failed to normalize enzymes or improve liver fat quantified by radiology imaging and other noninvasive measures. However, the limitations of these studies included not being adequately powered, with variable cutoffs for AHI to define OSA syndrome, variable diagnostic modalities for NAFLD and duration of CPAP therapy were rather too short. The longest duration of CPAP therapy among these studies was 3 months [14-17]. Another randomized study that evaluated the effect of CPAP treatment on liver fibrosis, using the FibroMax score (non-invasive testing called biopredictive tests that work by using an algorithm based on the patients' sex, age, weight, height and specific blood biomarkers) did not demonstrate any impact on reducing fibrosis; even though, it had more than 100 patients [16]. Contrary to the conclusions drawn from these randomized controlled trials, many observational and prospective cohort studies have associated adherence to CPAP therapy with improvement in liver enzymes and radiological steatosis [76-78]. Kim et al also concluded that there was a potential for reduction in liver fibrosis [79]. An important observation in all these studies was the duration of CPAP therapy which ranged from 1 to 3 years. A recent metaanalysis of 192 OSA syndrome patients showed that CPAP therapy for a minimum of 3 months was associated with statistically significant decrease in aspartate transaminase (AST) and alanine aminotransferase (ALT) [80]. One study by Chin et al with a sample size of 40 patients found that AST significantly decreased after single night CPAP therapy [81]. CPAP treatment significantly decreased the cumulative incidence as well as the overall risk for liver disease based on the large Taiwanese retrospective study with a follow-up duration of 10 years. This suggests that prolonged CPAP treatment plays a role in delaying progression and decreasing incidence of liver disease [82].

CPAP compliance was not adequately assessed in many RCTs looking into the event of CPAP therapy on NAFLD. This is one of the major limitations that inhibit any direct conclusion with respect to the effectiveness of CPAP therapy on resolution or reversal of NAFLD. CPAP compliance has varied definitions, for example, the Medicare criteria. In our practice, we find that the most beneficial outcomes are among patients with near maximum possible compliance. This, however, requires diligent and close long-term follow-up. Compliance among OSA syndrome patients in the real world has been shown to be around $20-50 \%$ [83-85]. Even by our practice standards, the definition of compliance used in these studies is suboptimal.

Unfortunately, the evidence to suggest positive outcomes of CPAP therapy among patients with liver disease has not been promising. Inadequate CPAP compliance and lack of long-term follow-up may account for the scarcity of evidence supporting positive outcomes of CPAP therapy among patients with NAFLD.

Our case has multiple important clinical implications. First, it is prudent to assume that OSA syndrome is associated with NAFLD. Although past studies have had contradicting results, they were flawed in design, non-randomized and small sized. More recent, better-designed studies have established a stronger link between the two. Second, a trial of treatment with CPAP in patients with OSA syndrome suspected to have NAFLD can help with diagnosis, treatment, follow-up and prognosis since definitive diagnosis of NASH requires a liver biopsy which is invasive and associated with complications. Third, treatment with CPAP must continue for a prolonged period of time as there is evidence to suggest that the beneficial effects of CPAP in liver disease need treatment adherence for a minimum of 3 months. Fourth, we recommend that every patient diagnosed with NAFLD should be screened for the presence of OSA. Fifth, we suggest the need for more RCTs assessing progression and potential reversal of NASH based on optimal compliance with CPAP therapy over significantly longer periods, as suggested by our case. Finally, CPAP treatments among patients who have OSA syndrome may be a pre- 
ventive measure for NAFLD.

\section{Conflict of Interest}

No conflict of interests to declare.

\section{Grant Support}

No grants received.

\section{Disclosure}

All authors have no financial disclosures.

\section{Informed Consent}

Informed consent obtained from the patient.

\section{References}

1. Liu X, Miao Y, Wu F, Du T, Zhang Q. Effect of CPAP therapy on liver disease in patients with OSA: a review. Sleep Breath. 2018.

2. Epstein LJ, Kristo D, Strollo PJ, Jr., Friedman N, Malhotra A, Patil SP, Ramar K, et al. Clinical guideline for the evaluation, management and long-term care of obstructive sleep apnea in adults. J Clin Sleep Med. 2009;5(3):263276.

3. The Report of an American Academy of Sleep Medicine Task Force. Sleep-related breathing disorders in adults: recommendations for syndrome definition and measurement techniques in clinical research. Sleep. 1999;22(5):667-689.

4. Vizuete J, Camero A, Malakouti M, Garapati K, Gutierrez J. Perspectives on Nonalcoholic Fatty Liver Disease: An Overview of Present and Future Therapies. J Clin Transl Hepatol. 2017;5(1):67-75.

5. Charlton M. Nonalcoholic fatty liver disease: a review of current understanding and future impact. Clin Gastroenterol Hepatol. 2004;2(12):1048-1058.

6. Abdelmalek MF, Diehl AM. Nonalcoholic fatty liver disease as a complication of insulin resistance. Med Clin North Am. 2007;91(6):1125-1149, ix.

7. West SD, Nicoll DJ, Stradling JR. Prevalence of obstructive sleep apnoea in men with type 2 diabetes. Thorax. 2006;61(11):945-950.

8. Coughlin SR, Mawdsley L, Mugarza JA, Calverley PM, Wilding JP. Obstructive sleep apnoea is independently associated with an increased prevalence of metabolic syndrome. Eur Heart J. 2004;25(9):735-741.

9. Marin JM, Carrizo SJ, Vicente E, Agusti AG. Long-term cardiovascular outcomes in men with obstructive sleep apnoea-hypopnoea with or without treatment with con- tinuous positive airway pressure: an observational study. Lancet. 2005;365(9464):1046-1053.

10. Drager LF, Polotsky VY, Lorenzi-Filho G. Obstructive sleep apnea: an emerging risk factor for atherosclerosis. Chest. 2011;140(2):534-542.

11. Younossi ZM, Stepanova M, Afendy M, Fang Y, Younossi Y, Mir H, Srishord M. Changes in the prevalence of the most common causes of chronic liver diseases in the United States from 1988 to 2008. Clin Gastroenterol Hepatol. 2011;9(6):524-530 e521; quiz e560.

12. Tanne F, Gagnadoux F, Chazouilleres O, Fleury B, Wendum D, Lasnier E, Lebeau B, et al. Chronic liver injury during obstructive sleep apnea. Hepatology. 2005;41(6):1290-1296.

13. Aron-Wisnewsky J, Minville C, Tordjman J, Levy P, Bouillot JL, Basdevant A, Bedossa P, et al. Chronic intermittent hypoxia is a major trigger for non-alcoholic fatty liver disease in morbid obese. J Hepatol. 2012;56(1):225233.

14. Hoyos CM, Killick R, Yee BJ, Phillips CL, Grunstein RR, Liu PY. Cardiometabolic changes after continuous positive airway pressure for obstructive sleep apnoea: a randomised sham-controlled study. Thorax. 2012;67(12):1081-1089.

15. Kohler M, Pepperell JC, Davies RJ, Stradling JR. Continuous positive airway pressure and liver enzymes in obstructive sleep apnoea: data from a randomized controlled trial. Respiration. 2009;78(2):141-146.

16. Kritikou I, Basta M, Tappouni R, Pejovic S, FernandezMendoza J, Nazir R, Shaffer ML, et al. Sleep apnoea and visceral adiposity in middle-aged male and female subjects. Eur Respir J. 2013;41(3):601-609.

17. Sivam S, Phillips CL, Trenell MI, Yee BJ, Liu PY, Wong KK, Grunstein RR. Effects of 8 weeks of continuous positive airway pressure on abdominal adiposity in obstructive sleep apnoea. Eur Respir J. 2012;40(4):913-918.

18. Lam JC, Lam B, Yao TJ, Lai AY, Ooi CG, Tam S, Lam $\mathrm{KS}$, et al. A randomised controlled trial of nasal continuous positive airway pressure on insulin sensitivity in obstructive sleep apnoea. Eur Respir J. 2010;35(1):138-145.

19. Jungermann K, Kietzmann T. Oxygen: modulator of metabolic zonation and disease of the liver. Hepatology. 2000;31(2):255-260.

20. Fandrey J, Gassmann M. Oxygen sensing and the activation of the hypoxia inducible factor 1 (HIF-1) - invited article. Adv Exp Med Biol. 2009;648:197-206.

21. Baze MM, Schlauch K, Hayes JP. Gene expression of the liver in response to chronic hypoxia. Physiol Genomics. 2010;41(3):275-288.

22. Choi JH, Park MJ, Kim KW, Choi YH, Park SH, An WG, Yang US, et al. Molecular mechanism of hypoxia-mediated hepatic gluconeogenesis by transcriptional regulation. FEBS Lett. 2005;579(13):2795-2801.

23. Nakagawa Y, Kishida K, Kihara S, Yoshida R, Funahashi $\mathrm{T}$, Shimomura I. Nocturnal falls of adiponectin levels in sleep apnea with abdominal obesity and impact of hypoxia-induced dysregulated adiponectin production in obese murine mesenteric adipose tissue. J Atheroscler Thromb. 2011;18(3):240-247. 
24. Nakagawa Y, Kishida K, Kihara S, Sonoda M, Hirata A, Yasui A, Nishizawa H, et al. Nocturnal reduction in circulating adiponectin concentrations related to hypoxic stress in severe obstructive sleep apnea-hypopnea syndrome. Am J Physiol Endocrinol Metab. 2008;294(4):E778-784.

25. Musso G, Olivetti C, Cassader M, Gambino R. Obstructive sleep apnea-hypopnea syndrome and nonalcoholic fatty liver disease: emerging evidence and mechanisms. Semin Liver Dis. 2012;32(1):49-64.

26. Singh H, Pollock R, Uhanova J, Kryger M, Hawkins K, Minuk GY. Symptoms of obstructive sleep apnea in patients with nonalcoholic fatty liver disease. Dig Dis Sci. 2005;50(12):2338-2343.

27. Tatsumi K, Saibara T. Effects of obstructive sleep apnea syndrome on hepatic steatosis and nonalcoholic steatohepatitis. Hepatol Res. 2005;33(2):100-104.

28. Kallwitz ER, Herdegen J, Madura J, Jakate S, Cotler SJ. Liver enzymes and histology in obese patients with obstructive sleep apnea. J Clin Gastroenterol. 2007;41(10):918-921.

29. Mishra P, Nugent C, Afendy A, Bai C, Bhatia P, Afendy M, Fang Y, et al. Apnoeic-hypopnoeic episodes during obstructive sleep apnoea are associated with histological nonalcoholic steatohepatitis. Liver Int. 2008;28(8):10801086.

30. Campos GM, Bambha K, Vittinghoff E, Rabl C, Posselt AM, Ciovica R, Tiwari U, et al. A clinical scoring system for predicting nonalcoholic steatohepatitis in morbidly obese patients. Hepatology. 2008;47(6):1916-1923.

31. Norman D, Bardwell WA, Arosemena F, Nelesen R, Mills PJ, Loredo JS, Lavine JE, et al. Serum aminotransferase levels are associated with markers of hypoxia in patients with obstructive sleep apnea. Sleep. 2008;31(1):121-126.

32. Polotsky VY, Patil SP, Savransky V, Laffan A, Fonti S, Frame LA, Steele KE, et al. Obstructive sleep apnea, insulin resistance, and steatohepatitis in severe obesity. Am J Respir Crit Care Med. 2009;179(3):228-234.

33. Turkay C, Ozol D, Kasapoglu B, Kirbas I, Yildirim Z, Yigitoglu R. Influence of obstructive sleep apnea on fatty liver disease: role of chronic intermittent hypoxia. Respir Care. 2012;57(2):244-249.

34. Tassone F, Lanfranco F, Gianotti L, Pivetti S, Navone F, Rossetto R, Grottoli S, et al. Obstructive sleep apnoea syndrome impairs insulin sensitivity independently of anthropometric variables. Clin Endocrinol (Oxf). 2003;59(3):374-379.

35. Yang D, Liu Z, Yang H, Luo Q. Effects of continuous positive airway pressure on glycemic control and insulin resistance in patients with obstructive sleep apnea: a meta-analysis. Sleep Breath. 2013;17(1):33-38.

36. Shehzad A, Iqbal W, Shehzad O, Lee YS. Adiponectin: regulation of its production and its role in human diseases. Hormones (Athens). 2012;11(1):8-20.

37. Hajer GR, van Haeften TW, Visseren FL. Adipose tissue dysfunction in obesity, diabetes, and vascular diseases. Eur Heart J. 2008;29(24):2959-2971.

38. Aurora RN, Punjabi NM. Obstructive sleep apnoea and type 2 diabetes mellitus: a bidirectional association. Lancet Respir Med. 2013;1(4):329-338.
39. Harsch IA, Schahin SP, Radespiel-Troger M, Weintz O, Jahreiss H, Fuchs FS, Wiest GH, et al. Continuous positive airway pressure treatment rapidly improves insulin sensitivity in patients with obstructive sleep apnea syndrome. Am J Respir Crit Care Med. 2004;169(2):156162.

40. Chen X, Niu X, Xiao Y, Dong J, Lu M, Kong W. Effect of continuous positive airway pressure on leptin levels in patients with obstructive sleep apnea: a meta-analysis. Otolaryngol Head Neck Surg. 2015;152(4):610-618.

41. Lin MT, Lin HH, Lee PL, Weng PH, Lee CC, Lai TC, Liu W, et al. Beneficial effect of continuous positive airway pressure on lipid profiles in obstructive sleep apnea: a meta-analysis. Sleep Breath. 2015;19(3):809-817.

42. Nadeem R, Singh M, Nida M, Kwon S, Sajid H, Witkowski J, Pahomov E, et al. Effect of CPAP treatment for obstructive sleep apnea hypopnea syndrome on lipid profile: a meta-regression analysis. J Clin Sleep Med. 2014;10(12):1295-1302.

43. Schulz R, Mahmoudi S, Hattar K, Sibelius U, Olschewski H, Mayer K, Seeger W, et al. Enhanced release of superoxide from polymorphonuclear neutrophils in obstructive sleep apnea. Impact of continuous positive airway pressure therapy. Am J Respir Crit Care Med. 2000;162(2 Pt 1):566-570.

44. Celec P, Hodosy J, Behuliak M, Palffy R, Gardlik R, Halcak L, Mucska I. Oxidative and carbonyl stress in patients with obstructive sleep apnea treated with continuous positive airway pressure. Sleep Breath. 2012;16(2):393-398.

45. Xie X, Pan L, Ren D, Du C, Guo Y. Effects of continuous positive airway pressure therapy on systemic inflammation in obstructive sleep apnea: a meta-analysis. Sleep Med. 2013;14(11):1139-1150.

46. Baessler A, Nadeem R, Harvey M, Madbouly E, Younus A, Sajid H, Naseem J, et al. Treatment for sleep apnea by continuous positive airway pressure improves levels of inflammatory markers - a meta-analysis. J Inflamm (Lond). 2013;10:13.

47. European Association for the Study of the Liver, European Association for the Study of Diabetes, European Association for the Study of Obesity. EASL-EASD-EASO clinical practice guidelines for the management of non-alcoholic fatty liver disease. Obes Facts. 2016;9(2):65-90.

48. Chalasani N, Younossi Z, Lavine JE, Diehl AM, Brunt EM, Cusi K, Charlton M, et al. The diagnosis and management of non-alcoholic fatty liver disease: practice guideline by the American Gastroenterological Association, American Association for the Study of Liver Diseases, and American College of Gastroenterology. Gastroenterology. 2012;142(7):1592-1609.

49. Araujo AR, Rosso N, Bedogni G, Tiribelli C, Bellentani S. Global epidemiology of non-alcoholic fatty liver disease/non-alcoholic steatohepatitis: What we need in the future. Liver Int. 2018;38(Suppl 1):47-51.

50. Brunt EM, Kleiner DE, Wilson LA, Belt P, Neuschwander-Tetri BA, Network NCR. Nonalcoholic fatty liver disease (NAFLD) activity score and the histopathologic diagnosis in NAFLD: distinct clinicopathologic meanings. Hepatology. 2011;53(3):810-820. 
51. Bedossa P, Poitou C, Veyrie N, Bouillot JL, Basdevant A, Paradis V, Tordjman J, et al. Histopathological algorithm and scoring system for evaluation of liver lesions in morbidly obese patients. Hepatology. 2012;56(5):1751-1759.

52. Ratziu V, Massard J, Charlotte F, Messous D, ImbertBismut F, Bonyhay L, Tahiri M, et al. Diagnostic value of biochemical markers (FibroTest-FibroSURE) for the prediction of liver fibrosis in patients with non-alcoholic fatty liver disease. BMC Gastroenterol. 2006;6:6.

53. Roulot D, Czernichow S, Le Clesiau H, Costes JL, Vergnaud AC, Beaugrand $\mathrm{M}$. Liver stiffness values in apparently healthy subjects: influence of gender and metabolic syndrome. J Hepatol. 2008;48(4):606-613.

54. Hamaguchi M, Kojima T, Itoh Y, Harano Y, Fujii K, Nakajima T, Kato T, et al. The severity of ultrasonographic findings in nonalcoholic fatty liver disease reflects the metabolic syndrome and visceral fat accumulation. Am J Gastroenterol. 2007;102(12):2708-2715.

55. Gastaldelli A, Kozakova M, Hojlund K, Flyvbjerg A, Favuzzi A, Mitrakou A, Balkau B, et al. Fatty liver is associated with insulin resistance, risk of coronary heart disease, and early atherosclerosis in a large European population. Hepatology. 2009;49(5):1537-1544.

56. Chen X, Lin X, Chen LD, Lin QC, Chen GP, Yu YH, Huang JC, et al. Obstructive sleep apnea is associated with fatty liver index, the index of nonalcoholic fatty liver disease. Eur J Gastroenterol Hepatol. 2016;28(6):650655.

57. Cohen JC, Horton JD, Hobbs HH. Human fatty liver disease: old questions and new insights. Science. 2011;332(6037):1519-1523.

58. Musso G, Gambino R, Cassader M, Pagano G. Metaanalysis: natural history of non-alcoholic fatty liver disease (NAFLD) and diagnostic accuracy of non-invasive tests for liver disease severity. Ann Med. 2011;43(8):617649.

59. Romero E, Krakow B, Haynes P, Ulibarri V. Nocturia and snoring: predictive symptoms for obstructive sleep apnea. Sleep Breath. 2010;14(4):337-343.

60. Antic NA, Catcheside P, Buchan C, Hensley M, Naughton MT, Rowland S, Williamson B, et al. The effect of CPAP in normalizing daytime sleepiness, quality of life, and neurocognitive function in patients with moderate to severe OSA. Sleep. 2011;34(1):111-119.

61. Osman AM, Carter SG, Carberry JC, Eckert DJ. Obstructive sleep apnea: current perspectives. Nat Sci Sleep. 2018;10:21-34.

62. Ye L, Pien GW, Ratcliffe SJ, Bjornsdottir E, Arnardottir ES, Pack AI, Benediktsdottir B, et al. The different clinical faces of obstructive sleep apnoea: a cluster analysis. Eur Respir J. 2014;44(6):1600-1607.

63. Arnardottir ES, Bjornsdottir E, Olafsdottir KA, Benediktsdottir B, Gislason T. Obstructive sleep apnoea in the general population: highly prevalent but minimal symptoms. Eur Respir J. 2016;47(1):194-202.

64. Bedossa P. Diagnosis of non-alcoholic fatty liver disease/ non-alcoholic steatohepatitis: Why liver biopsy is essential. Liver Int. 2018;38(Suppl 1):64-66.

65. Mulgrew AT, Fox N, Ayas NT, Ryan CF. Diagnosis and initial management of obstructive sleep apnea without polysomnography: a randomized validation study. Ann Intern Med. 2007;146(3):157-166.

66. Magalang UJ, Dmochowski J, Veeramachaneni S, Draw A, Mador MJ, El-Solh A, Grant BJ. Prediction of the apnea-hypopnea index from overnight pulse oximetry. Chest. 2003;124(5):1694-1701.

67. Cheliout-Heraut F, Senny F, Djouadi F, Ouayoun M, Bour F. Obstructive sleep apnoea syndrome: comparison between polysomnography and portable sleep monitoring based on jaw recordings. Neurophysiol Clin. 2011;41(4):191-198.

68. Kapur VK, Auckley DH, Chowdhuri S, Kuhlmann DC, Mehra R, Ramar K, Harrod CG. Clinical practice guideline for diagnostic testing for adult obstructive sleep apnea: an American Academy of Sleep Medicine Clinical Practice Guideline. J Clin Sleep Med. 2017;13(3):479504.

69. Dyson JK, Anstee QM, McPherson S. Republished: Nonalcoholic fatty liver disease: a practical approach to treatment. Postgrad Med J. 2015;91(1072):92-101.

70. Dyson J, Day C. Treatment of non-alcoholic fatty liver disease. Dig Dis. 2014;32(5):597-604.

71. Neuschwander-Tetri BA. Non-alcoholic fatty liver disease. BMC Med. 2017;15(1):45.

72. Sharma SK, Agrawal S, Damodaran D, Sreenivas V, Kadhiravan T, Lakshmy R, Jagia P, et al. CPAP for the metabolic syndrome in patients with obstructive sleep apnea. N Engl J Med. 2011;365(24):2277-2286.

73. Abe H, Takahashi M, Yaegashi H, Eda S, Tsunemoto H, Kamikozawa M, Koyama J, et al. Efficacy of continuous positive airway pressure on arrhythmias in obstructive sleep apnea patients. Heart Vessels. 2010;25(1):63-69.

74. Drager LF, Bortolotto LA, Figueiredo AC, Krieger EM, Lorenzi GF. Effects of continuous positive airway pressure on early signs of atherosclerosis in obstructive sleep apnea. Am J Respir Crit Care Med. 2007;176(7):706-712.

75. Shaw JE, Punjabi NM, Wilding JP, Alberti KG, Zimmet PZ. International Diabetes Federation Taskforce on E, Prevention. Sleep-disordered breathing and type 2 diabetes: a report from the International Diabetes Federation Taskforce on Epidemiology and Prevention. Diabetes Res Clin Pract. 2008;81(1):2-12.

76. Shpirer I, Copel L, Broide E, Elizur A. Continuous positive airway pressure improves sleep apnea associated fatty liver. Lung. 2010;188(4):301-307.

77. Hobzova M, Ludka O, Stepanova R, Sovova E. Continuous positive airway pressure treatment and liver enzymes in sleep apnea patients. Sleep Medicine. 2015;16:S215S216.

78. Buttacavoli M, Gruttad'Auria CI, Olivo M, Virdone R, Castrogiovanni A, Mazzuca E, Marotta AM, et al. Liver Steatosis and Fibrosis in OSA patients After Long-term CPAP Treatment: A Preliminary Ultrasound Study. Ultrasound Med Biol. 2016;42(1):104-109.

79. Kim D, Mannalithara A, Udompap P, Kushida C, Ray Kim W. Impact of continuous positive airway pressure treatment on non-alcoholic fatty liver disease in patients with obstructive sleep apnea. Journal of Hepatology. 
2016;64(2):S479-S480.

80. Chen LD, Lin L, Zhang LJ, Zeng HX, Wu QY, Hu MF, Xie JJ, et al. Effect of continuous positive airway pressure on liver enzymes in obstructive sleep apnea: A metaanalysis. Clin Respir J. 2018;12(2):373-381.

81. Chin K, Nakamura T, Takahashi K, Sumi K, Ogawa Y, Masuzaki H, Muro S, et al. Effects of obstructive sleep apnea syndrome on serum aminotransferase levels in obese patients. Am J Med. 2003;114(5):370-376.

82. Hang LW, Chen CF, Wang CB, Wu TN, Liang WM, Chou TC. The association between continuous positive airway pressure therapy and liver disease development in obstructive sleep apnea/hypopnea syndrome patients: a nationwide population-based cohort study in Taiwan. Sleep
Breath. 2017;21(2):461-467.

83. Wolkove N, Baltzan M, Kamel H, Dabrusin R, Palayew $\mathrm{M}$. Long-term compliance with continuous positive airway pressure in patients with obstructive sleep apnea. Can Respir J. 2008;15(7):365-369.

84. Lee CHK, Leow LC, Song PR, Li H, Ong TH. Acceptance and adherence to continuous positive airway pressure therapy in patients with Obstructive Sleep Apnea (OSA) in a Southeast Asian privately funded healthcare system. Sleep Sci. 2017;10(2):57-63.

85. Choi J-A, Yoon I-Y, Han E-G, Lee S. Subjective and Objective CPAP Compliance in patients with obstructive sleep apnea syndrome. Sleep Medicine Research. 2011;2(2):63-68. 